\title{
Effect of modulation on radiation-induced instability
}

\author{
WEN-MEI YANG \\ Department of Mechanical Engineering, National Chiao Tung University, Hsinchu, Taiwan 30050, \\ Republic of China
}

(Received 4 August 1993 and in final form 13 May 1994)

\begin{abstract}
The instability of a fluid layer induced by modulated irradiation is studied numerically. Based on the Eddington approximation for the equation of transfer, the time-periodic temperature profile of the basic state is solved analytically. A system of linear equations with periodic coefficients describing the behavior of disturbances is obtained by linear stability theory. Using Floquet's theory, the disturbances are expanded by a double series of mixed Fourier and Chebyshev form. An algorithm combining Galerkin and collocation methods is developed and successfully traces the stability boundary between stable and transiently stable states. For the case of a fluid layer heated from below by a modulated temperature, the results show that modulation has a destabilizing effect at low frequencies and a stabilizing effect at high frequencies, which is in agreement with the available theoretical analyses and experimental data. The effects of Biot number and radiative parameters, such as Planck number and optical thickness, on the critical Rayleigh number are analyzed and compared with the unmodulated cases.
\end{abstract}

\section{INTRODUCTION}

Radiation-induced flow is an important phenomenon in meteorology and has recently been noticed in the technologies of laser fusion, material processing, and collection and utilization of solar energy. Because of the absorbing, emitting and scattering behavior of the participating media, the heat transfer and fluid flow are strongly affected by the radiative properties of the media and the boundaries, the geometry of system, and the magnitude of irradiation. Webb and Viskanta [1] theoretically and experimentally studied the natural convection in a vertical rectangular enclosure heated by irradiation from one side. Yang [2] investigated the stability of a horizontal fluid layer irradiated from the top and obtained the criteria for the onset of instability. Recently, Yang and Leu [3] further found the criteria of onset of secondary flow in an inclined slot irradiated from one side.

The effect of modulation on the dynamic system has long been interesting because stabilization or destabilization may occur in the presence of modulation, which thus enhances the mass, momentum and heat transport. The effects of modulation on the Benard instability were studied by Venezian [4], Rosenblat and Herbert [5], Rosenblat and Tanaka [6], Yih and Li [7], Gresho and Sani [8], and Finucane and Kelly [9]; and on the Taylor instability by Donnelly [10], and Donnelly et al. [11]. A review of papers on this subject can be found in the article by Davis [12]. It is commonly termed a basic periodic state, unstable if there exists a disturbance that experiences net growth over each modulation cycle, stable if every disturbance decays at every instant, or transiently stable if a disturbance grows during part of the cycle but ultimately decays. Among those studies, Rosenblat and Herbert [5] considered a fluid layer of freefree boundaries which is heated from below. By expanding the variables in frequency power series they obtained the periodicity criterion for which the basic state is unstable if an infinitesimal disturbance achieves net growth over one cycle of oscillation. From another viewpoint, the amplitude criterion was proposed for which the basic state is unstable if any disturbance increases during the cycle by an arbitrary factor of 10 . They concluded that the periodicity criterion is a sufficient condition for instability and the amplitude criterion can be regarded as a sufficient condition for stability. It was also found that a transiently stable state exists between these two critical conditions at low frequencies. On the other hand, Rosenblat and Tanaka [6] numerically showed that the critical Rayleigh number for the case of rigid-rigid boundaries always experiences positive enhancement in the presence of a periodic modulation which is consistent with the periodicity criterion. Finucane and Kelly [9] later found both experimentally and analytically that for low frequencies the modulation is destabilizing, whereas at higher frequencies stabilization is apparent. Their results support the amplitude criterion at low frequencies and periodicity criterion at $\omega>3$. In the present work, a numerical method is developed to trace the stability boundary between stable and transiently stable states and is justified by comparison with known results. Then the onset of convection of a fluid layer induced by modulated irradiation is considered. The effects of ampli- 


\section{NOMENCLATURE}

$\begin{array}{ll}A & \text { constant }\left(=3 \tau^{2}\right) \\ B & \text { constant }(=4 \eta \tau P) \\ B i & \text { Biot number } \\ \boldsymbol{g} & \text { gravity } \\ h & \text { convective heat transfer coefficient } \\ J & \text { magnitude of the disturbance of } j \\ j & \text { zeroth moment of radiative intensity } \\ k & \text { wavenumber } \\ L & \text { thickness of a fluid layer } \\ P & \text { Planck number } \\ p & \text { pressure } \\ P r & \text { Prandtl number } \\ \mathbf{q} & \text { complex radiative flux } \\ q_{3}^{\mathrm{R}} & \text { radiative heat flux } \\ q^{\prime \prime} & \text { irradiation, constant part } \\ R a & \text { Rayleigh number } \\ T & \text { dimensional temperature } \\ T_{n} & \text { nth degree Chebyshev polynomial } \\ t & \text { time } \\ V & \text { magnitude of the disturbance of } v_{3} \\ v_{i} & \text { velocities } \\ x_{i} & \text { coordinates. }\end{array}$

$\begin{array}{ll}\alpha_{R} & \text { Rosseland mean absorption coefficient } \\ \varepsilon & \text { amplitude of modulation } \\ \Phi & \text { magnitudes of disturbances } \\ \phi^{\prime} & \text { disturbances } \\ \zeta & \text { coordinate for Chebyshev polynomials } \\ \eta & \text { non-grayness } \\ \Theta & \text { magnitude of the disturbance of } \theta \\ \theta & \text { complex temperature } \\ \theta & \text { temperature } \\ \kappa & \text { thermal conductivity } \\ \mu & \text { dynamic viscosity } \\ \nu & \text { kinematic viscosity } \\ \sigma & \text { Stefan-Boltzmann constant or growth } \\ & \text { rate of the disturbances } \\ \tau & \text { optical thickness } \\ \Omega & \text { dimensional frequency of modulation } \\ \omega & \text { frequency of modulation. }\end{array}$

Superscript

- basic state.
Subscripts
c critical value of unmodulated case
p periodic
$\mathrm{s}$ non-periodic
$\infty \quad$ surrounding.

Greek symbols
$\alpha \quad$ thermal diffusivity
$\alpha_{P} \quad$ Planck mean absorption coefficient

tude and frequency of modulation as well as the radiative properties on the onset of instability are examined.

\section{PHYSICAL SYSTEM AND THE BASIC STATE}

Consider a horizontal layer of fluid of thickness $L$ placed above a solid boundary and subjected to a modulated irradiation $q^{\prime \prime}(1+\varepsilon \cos \Omega t)$ from the upper free surface, as shown in Fig. 1. The lower solid boundary is assumed to be insulated and black. The upper free surface is assumed to be transparent and

$$
q^{\prime \prime}(1+\varepsilon \cos \Omega t)
$$

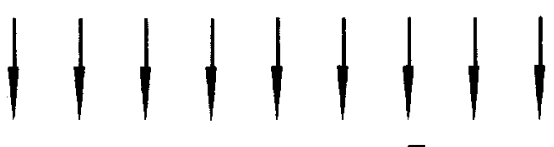

h $\quad \mathrm{T}_{\infty}$

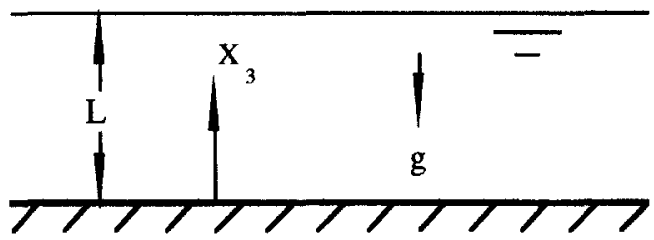

Fig. 1. A fluid layer heated by modulated irradiation. has a convective heat transfer coefficient $h$ with the surroundings, which is at a temperature of $T_{x}$.

For the incompressible fluids with constant properties and satisfying the Boussinesq approximation, using Eddington approximation for the transfer equation, the equations governing the flow and heat transfer in the participating media can be referred to Yang [2], and Arpaci and Gozum [13]. In this paper the dimensionless quantities are defined in the same way as those in Yang [2], while only the nonscattering cases are considered.

In the basic state, the fluid is stationary. By linearizing the emissive power, the one-dimensional equations describing the periodic temperature and radiative heat flux are

$$
\begin{gathered}
\frac{\partial \bar{\theta}}{\partial t}=\frac{\partial^{2} \bar{\theta}}{\partial x_{3} \partial x_{3}}-\frac{\partial \bar{q}_{3}^{\mathrm{R}}}{\partial x_{3}} \\
\frac{\partial^{2} \bar{q}_{3}^{\mathrm{R}}}{\partial x_{3} \partial x_{3}}-3 \tau^{2} \tilde{q}_{3}^{\mathrm{R}}=4 \eta \tau P \frac{\partial \bar{\theta}}{\partial x_{3}}
\end{gathered}
$$

where $\tau=\left(\alpha_{p} \alpha_{R}\right)^{1 / 2} L$ is the optical thickness, $\alpha_{p}$ and $\alpha_{R}$ are the Planck and the Rosseland mean absorption coefficients, respectively, $\eta=\left(\alpha_{p} / \alpha_{R}\right)^{1 / 2}$ is the nongrayness, $P=4 \sigma T_{\infty}^{3} /(\kappa / L)$ is the Planck number, and $\sigma$ is the Stefan-Boltzmann constant. The associated boundary conditions are

$$
-\frac{\mathrm{d} \bar{\theta}}{\mathrm{d} x_{3}}+\bar{q}_{3}^{\mathrm{R}}=0 \quad \text { at } x_{3}=0
$$




$$
\begin{gathered}
\frac{1}{\eta \tau} \frac{\mathrm{d} \bar{q}_{3}^{\mathrm{R}}}{\mathrm{d} x_{3}}-2 \bar{q}_{3}^{\mathrm{R}}=0 \quad \text { at } x_{3}=0 \\
-\frac{\mathrm{d} \bar{\theta}}{\mathrm{d} x_{3}}=B i\left(\bar{\theta}-\theta_{\infty}\right) \quad \text { at } x_{3}=1 \\
\frac{1}{\eta \tau} \frac{\mathrm{d} \bar{q}_{3}^{\mathrm{R}}}{\mathrm{d} x_{3}}+2 \bar{q}_{3}^{\mathrm{R}}=4\left[P\left(\bar{\theta}-\theta_{\infty}\right)-(1+\varepsilon \cos \omega t)\right] \\
\text { at } x_{3}=1
\end{gathered}
$$

where $B i=h L / \kappa$ is the Biot number, and $\omega=\Omega L^{2} / \alpha$ is the dimensionless angular frequency of modulation.

Since the basic-state solutions are periodic, they can be separated into two parts,

$$
\begin{gathered}
\bar{\theta}-\theta_{\infty}=\bar{\theta}_{\mathrm{s}}+\bar{\theta}_{\mathrm{p}} \\
\bar{q}_{3}^{\mathrm{R}}=\bar{q}_{\mathrm{s}}^{\mathrm{R}}+\bar{q}_{\mathrm{p}}^{\mathrm{R}}
\end{gathered}
$$

where the subscripts $\mathrm{s}$ and $\mathrm{p}$ represent nonperiodic and periodic, respectively. The nonperiodic part has been solved by Yang [2], and Yang and Leu [3], and the periodic part can be solved by letting

$$
\begin{aligned}
\bar{\theta}_{\mathrm{p}} & =\operatorname{Real}\left\{\theta \mathrm{e}^{i \omega t}\right\} \\
\bar{q}_{\mathrm{p}}^{\mathrm{R}} & =\operatorname{Real}\left\{\boldsymbol{q} \mathrm{e}^{i \omega t}\right\} .
\end{aligned}
$$

Here $\theta$ and $q$ are two complex functions of $x_{3}$ satisfying

$$
\begin{gathered}
\left(D^{2}-i \omega\right) \boldsymbol{\theta}-D \mathbf{q}=0 \\
-B D \boldsymbol{\theta}+\left(D^{2}-A\right) \mathbf{q}=0
\end{gathered}
$$

where $D=\mathrm{d} / \mathrm{d} x_{3}, A=3 \tau^{2}$ and $B=4 \eta \tau P$. The boundary conditions are

$$
\begin{gathered}
D \boldsymbol{\theta}-\mathbf{q}=0 \quad \text { at } x_{3}=0 \\
\frac{1}{\eta \tau} D \mathbf{q}-2 \mathbf{q}=0 \quad \text { at } x_{3}=0 \\
D \boldsymbol{\theta}+B i \boldsymbol{\theta}=0 \quad \text { at } x_{3}=1 \\
\frac{1}{\eta \tau} D \mathbf{q}+2 \mathbf{q}-4 P \boldsymbol{\theta}=-4 \varepsilon \quad \text { at } x_{3}=1 .
\end{gathered}
$$

The general solutions of $\boldsymbol{\theta}$ and $\mathbf{q}$ can be written as

$$
\begin{array}{r}
\boldsymbol{\theta}=c_{1} \cosh \left(\lambda_{1} x_{3}\right)+c_{2} \sinh \left(\lambda_{1} x_{3}\right) \\
+c_{3} \cosh \left(\lambda_{2} x_{3}\right)+c_{4} \sinh \left(\lambda_{2} x_{3}\right) \\
\mathbf{q}=c_{1} \frac{\lambda_{1}^{2}-i \omega}{\lambda_{1}} \sinh \left(\lambda_{1} x_{3}\right)+c_{2} \frac{\lambda_{1}^{2}-i \omega}{\lambda_{1}} \cosh \left(\lambda_{1} x_{3}\right) \\
+c_{3} \frac{\lambda_{2}^{2}-i \omega}{\lambda_{2}} \sinh \left(\lambda_{2} x_{3}\right)+c_{4} \frac{\lambda_{2}^{2}-i \omega}{\lambda_{2}} \cosh \left(\lambda_{2} x_{3}\right) .
\end{array}
$$

In the above equations, $c_{1}, c_{2}, c_{3}$ and $c_{4}$ can be determined from the boundary conditions (10), and $\pm \lambda_{1}$ and $\pm \lambda_{2}$ are the roots of the characteristic equation

$$
\lambda^{4}-(A+B+i \omega) \lambda^{2}+i \omega A=0 .
$$

\section{LINEAR STABILITY ANALYSIS}

In the linear stability analysis, the perturbed quantities are added to the basic state, and then all nonlinear terms are neglected. Because the periodic behavior of the basic state appears in the coefficients of disturbance equations, the perturbed quantities, according to the normal mode analysis and Floquet's theory $[14,15]$, can be expressed by

$$
\phi^{\prime}=\Phi\left(x_{3}, t\right) \mathrm{e}^{i\left(k_{1} x_{1}+k_{2} \cdot x_{2}\right)+\sigma t} .
$$

Here $\phi^{\prime}$ represents the disturbances, $\Phi$ is the magnitude of disturbances and is periodic with the same period as that of the basic state, $k_{1}$ and $k_{2}$ are the wavenumbers of the disturbances in the $x_{1}$ and $x_{2}$ directions, respectively, and $\sigma=\sigma_{\mathrm{r}}+i \sigma_{i}$ is the growth rate of the disturbances. The basic state, with respect to the infinitesimal disturbances, is unstable if $\sigma_{\mathrm{r}}$ is greater than zero or stable if $\sigma_{\mathrm{r}}$ is less than zero. Here unstable means that an infinitesimal disturbance either achieves net growth over one cycle of modulation, or grows during part of the cycle but ultimately decays. At the neutral stable state $\sigma_{r}$ is equal to zero.

With some similar manipulations as Yang [2], the equations describing the disturbances can be obtained :

$$
\begin{gathered}
-\frac{1}{\operatorname{Pr}}\left(D^{2}-k^{2}\right) \frac{\partial V}{\partial t}+\left(D^{2}-k^{2}\right)^{2} V-k^{2} R a \Theta \\
=\frac{\sigma}{P r}\left(D^{2}-k^{2}\right) V \\
-\frac{\partial \Theta}{\partial t}-\frac{\partial \bar{\theta}}{\partial x_{3}} V+\left(D^{2}-k^{2}-4 \eta \tau P\right) \Theta+\eta \tau J=\sigma \Theta
\end{gathered}
$$

where $V, \Theta$ and $J$ are the magnitudes of the disturbances for the $x_{3}$-component velocity, temperature and radiation, respectively, $P r=v / \alpha$ is the Prandt number, $R a=\left(\boldsymbol{g} \beta L^{3} / v \alpha\right)\left(q^{\prime \prime} L / \kappa\right)$ is the Rayleigh number, $\beta$ the thermal expansion coefficient, $D=\mathrm{d} / \mathrm{d} x_{3}$ and $k^{2}=k_{1}^{2}+k_{2}^{2}$. The associated boundary conditions are

$$
\begin{gathered}
V=D V=0 \quad \text { at } x_{3}=0 \\
D \Theta+\frac{\eta}{3 \tau} D J=0 \quad \text { at } x_{3}=0 \\
4 P \Theta+\frac{2 \eta}{3 \tau} D J-J=0 \quad \text { at } x_{3}=0 \\
V=D^{2} V=0 \quad \text { at } x_{3}=1 \\
D \Theta+B i \Theta=0 \quad \text { at } x_{3}=1 \\
\frac{2 \eta}{3 \tau} D J+J=0 \quad \text { at } x_{3}=1 .
\end{gathered}
$$

Equations (15)-(17) with the boundary conditions (18) constitute an eigenvalue problem. For the existence of nontrivial solutions, the eigenvalues $\sigma$ are of an infinitely great number of discrete values and 
depend on the parameters and the wavenumber functionally as

$$
\sigma=f(R a, P r, B i, P, \eta, \tau, \varepsilon, \omega, k) .
$$

At the neutral state, the real part of the most unstable eigenvalue vanishes. The critical Rayleigh number occurs at a neutral state which has a minimum Rayleigh number at the corresponding critical wavenumber.

To solve the linear differential eigensystem (15)(18), the unknowns are expanded, after the transformation $\zeta=2\left(x_{3}-1 / 2\right)$ is made, by the combination of complex Fourier series and Chebyshev polynomials :

$$
\begin{gathered}
V=\sum_{m=-\infty}^{\infty} \sum_{n=2}^{\infty} a_{m n}\left\{T_{n}-\frac{1}{2}\left[1-(-1)^{n}\right] T_{1}\right. \\
\left.-\frac{1}{2}\left[1+(-1)^{n}\right] T_{0}\right\} \mathrm{e}^{i m(v)} \\
\Theta=\sum_{m=-\infty}^{\infty} \sum_{n=0}^{\infty} b_{m n} T_{n} \mathrm{e}^{i m \omega t} \\
J=\sum_{m=-\infty}^{\infty} \sum_{n=0}^{\infty} c_{m n} T_{n} \mathrm{e}^{i m \omega n t}
\end{gathered}
$$

where $a_{m n}, b_{m n}$ and $c_{m n}$ are unknown coefficients, and $T_{n}(\zeta)$ is the $n$th degree Chebyshev polynomial of the first kind [16], defined in the interval $-1 \leqslant \zeta \leqslant 1$. The reason to choose the trial functions, which satisfy only two boundary conditions for $V$ but none for $\Theta$ and $J$, was explained by Yang [2].

Substituting the lowest $M \times N$ terms of equations (20)-(22) into equations (15)-(17), then eliminating $t$ by the Galerkin method, i.e. multiplying $\mathrm{e}^{-i m o s}$ then integrating over one period, and requesting the identities to hold at the $N-2$ collocation points,

$$
\zeta_{n}=\cos \left(\frac{n}{N-1}\right) \pi \quad n=1,2, \ldots, N-2
$$

and at the two boundary conditions for each variable, an algebraic eigensystem is obtained:

$$
A X=\sigma B X \text {. }
$$

Here, $A$ and $B$ are two $3(M \times N) \times 3(M \times N)$ coefficient matrices. $X$ is a $3(M \times N)$ vector composed of the unknown coefficients.

The eigenvalues of the generalized eigensystem (23) is solved by the $Q Z$ algorithm [17], which traces all eigenvalues with high accuracy. By examining the structure of equation (23), it is found that $c_{m n}$ can be eliminated without influencing the eigenvalues. A condensed eigensystem of order $2(M \times N)$ is obtained for reducing computation time, before the eigenvalue solver is called. The $R a$ at the neutral stable state, at which the real part of the most unstable eigenvalue vanishes, is searched by the secant method, which needs two initial guesses. The iteration is not terminated until the real part of the most unstable eigenvalue is less than $10^{-6}$. For the fixed parameters, different $R a$ at neutral stable states may be obtained

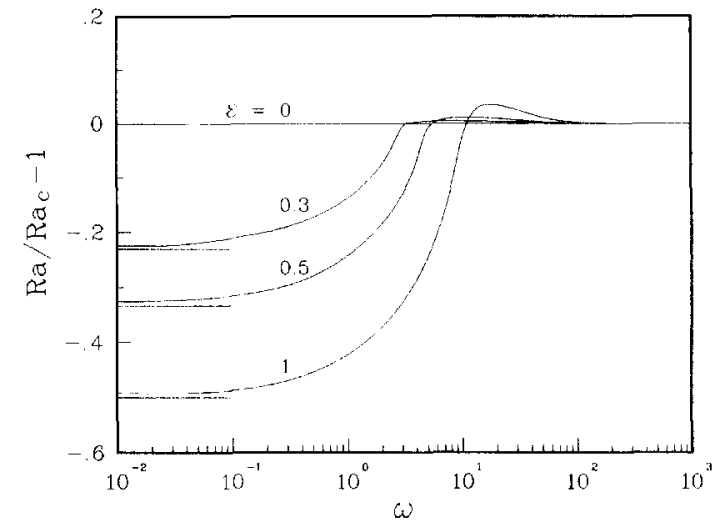

Fig. 2. Percentage change of critical Rayleigh number vs frequency of a fluid layer with $\operatorname{Pr}=0.7$ sinusoidally heated from below.

for different wavenumbers. The minimum Rayleigh number is called the critical Rayleigh number, which occurs at the critical wavenumber.

To assure the validity of the above algorithm, the case of a fluid layer between two rigid boundaries, with a fixed temperature at the upper boundary and a modulated temperature at the lower boundary, is calculated and compared with the available results $[5$, 9]. The cases for $\operatorname{Pr}=0.7$ and $\varepsilon=0,0.3,0.5$ and 1 are shown in Fig. 2 where the ordinate is in terms of the percentage change of the critical Rayleigh number, compared with the unmodulated case $R a_{\mathrm{c}}=1707.76$. At low frequencies, it is seen that modulation has a destabilizing effect, which is consistent with the amplitude criterion of Rosenblat and Herbert [5]. In the quasi-steady limit $\omega \rightarrow 0$, the percentage change of the critical Rayleigh number is, within a certain accuracy, close to the theoretical result $\left(R a / R a_{\mathrm{c}}\right)-1 \rightarrow-\varepsilon /$ $(1+\varepsilon)$, which is expressed by the horizontal lines below each curve. At high frequencies, modulation has a stabilizing effect which is consistent with the periodicity criterion of Rosenblat and Herbert [5], for which instability occurs globally and can be observed over one cycle. The destabilization effect at low frequencies, as well as the stabilization effect for $\omega=3.5$ and $0.8<\varepsilon<1$, was experimentally approved for air by Finucane and Kelly [9]. In the present calculation the stabilization is found to occur starting from $\omega \approx 3$ for $\varepsilon=0.3$ and $\omega \approx 10$ for $\varepsilon=1$, which is in broad agreement with the experiment.

\section{RESULTS AND DISCUSSION}

To simplify the analysis of the complicated relation of equation (23), $\eta$ is set equal to 1 , which means that the participating medium is assumed to be gray. The convergence of numerical results is examined by using different resolutions to calculate the critical Rayleigh number for a typical case. The results for $\operatorname{Pr}=0.7$, $B i=1, P=1, \tau=1, \varepsilon=1, \omega=0.01$, and $k=2.12$ 
Table 1. Ra vs $M$ and $N$ for $\operatorname{Pr}=0.7, B i=1, P=1, \tau=1$, $\varepsilon=1, \omega=0.01$ and $k=2.12$

\begin{tabular}{rccc}
$M$ & \multicolumn{3}{c}{$N$} \\
& 6 & 8 & 10 \\
\hline 3 & 2878.62 & 2914.61 & 2913.88 \\
5 & 2633.50 & 2666.43 & 2665.76 \\
7 & 2554.40 & 2586.34 & 2585.70 \\
9 & 2519.03 & 2550.52 & 2549.89 \\
11 & 2500.34 & 2531.60 & 2530.97 \\
13 & 2489.55 & 2520.68 & 2520.05 \\
15 & 2483.14 & 2514.18 & 2513.56 \\
\hline
\end{tabular}

are shown in Table 1 for various combinations of $M$ and $N$. It is seen that at a fixed $M$ the critical Rayleigh number converges rapidly as $N$ is increased from 8 to 10 , which shows the exponential convergence of the Chebyshev polynomials, whereas a larger $M$ than $N$ is needed for the expansion in time by a complete set of Fourier series. In the following calculations, the results are obtained from two consecutive resolutions for which the relative variation is less than $1 \%$ and the error for the case of quasi-steady limit, $\omega \rightarrow 0$, compared with the theoretical value $\left(R a / R a_{\mathrm{c}}\right)-1 \rightarrow$ $-\varepsilon /(1+\varepsilon)$ is within $2 \%$.

When radiation is considered in thermal instability with fixed boundary temperatures, it was found [13] that radiation is an additional mode of heat transfer besides conduction, thus enhancing fluid particles to release energy and delaying the onset of instability. While for the situation of radiation-induced instability [2], because the extinction effect of participating media on the irradiation is proportional to the optical thickness, the onset of instability occurs at a higher critical Rayleigh number for larger optical thickness than for smaller optical thickness. When the irradiation is modulating, the frequency has a similar effect to a layer of fluid heated from below by a modulated temperature. As shown in Fig. 3 for $\operatorname{Pr}=0.7, B i=1$, $P=1$, and $\tau=1$, at low frequencies, modulation has a destabilizing effect which increases with increasing amplitude of modulation. The horizontal lines below

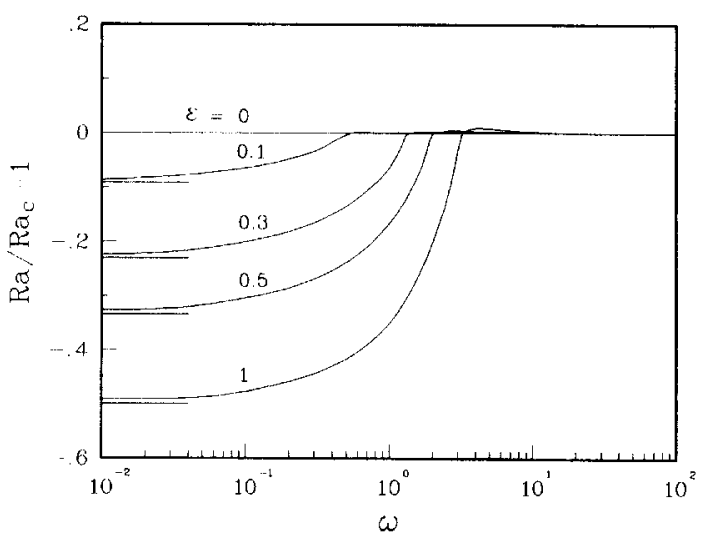

Fig. 3. Percentage change of critical Rayleigh number vs frequency for $\operatorname{Pr}=0.7, B i=1, P=1$, and $\tau=1$.

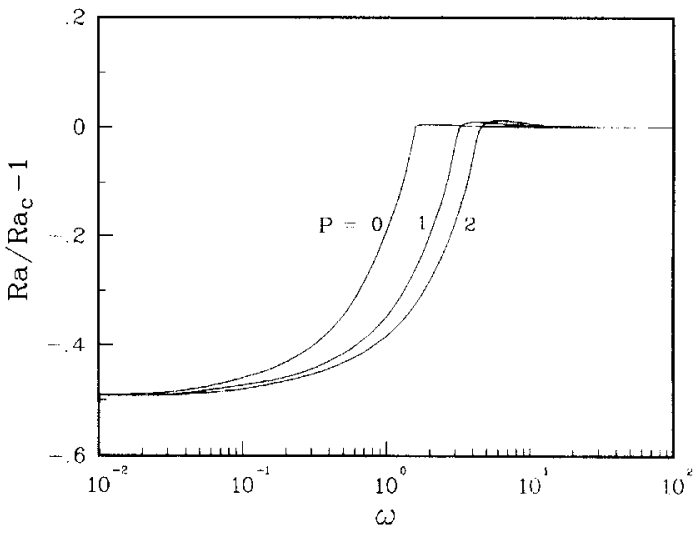

Fig. 4. Percentage change of critical Rayleigh number vs frequency at different Planck numbers for $\operatorname{Pr}=0.7, B i=1$, $\tau=1$, and $\varepsilon=1$.

each curve represent the quasi-steady limit $\omega \rightarrow 0$ for which $\left(R a / R a_{\mathrm{c}}\right)-1 \rightarrow-\varepsilon /(1+\varepsilon)$, where $R a_{\mathrm{c}}$ is the critical Rayleigh number for the unmodulated cases, and can be found in ref. [2]. As frequency increases in the intermediate range, the critical Rayleigh number increases rapidly and becomes slightly higher than the value of unmodulation at a certain value for each amplitude. For frequencies higher than this value, modulation has a slight stabilizing effect, which reaches a maximum and then gradually decreases as $\omega$ continually increases. By comparing Figs. 2 and 3 . it is seen that the stabilizing effect started at a lower frequency and the maximum effect is smaller for the case of radiation-induced flow (Fig. 3) than for the case of free radiation (Fig. 2).

The Planck number, the ratio of emission to conduction, is an important parameter in radiation heat transfer. When $P=0$, emission in the fluid layer is negligible compared with conduction. Consequently, the temperature distribution is linear and the critical Rayleigh number is smaller than the cases with a nonzero Planck number [2]. In the modulated cases, the smallest critical Rayleigh number still occurs at $P=0$. But, in terms of percentage change of Rayleigh number, as shown in Fig. 4 for $P r=0.7, B i=1, \tau=1$, and $\varepsilon=1$, with $P=0,1$ and 2 , it can be seen that for smaller Planck number, the stabilizing effect starts at a lower frequency and the effect increases with increasing Planck number.

Optical thickness, the ratio of the thickness of fluid layer to the penetration depth of radiation, is another important parameter in radiation-induced instability. Basically, the larger the optical thickness, the more radiative energy is retarded in reaching the lower boundary. Increasing optical thickness delays the onset of instability, and this is especially apparent for $\tau \geqslant 1$ [2]. For modulation being present, the percentage change of Rayleigh number is shown in Fig. 5 for $\tau=0.01,0.1$ and 1, with $\operatorname{Pr}=0.7, B i=1, P=1$, and $\varepsilon=1$. It is seen that the destabilization occurs at a lower frequency range for smaller optical thicknesses 


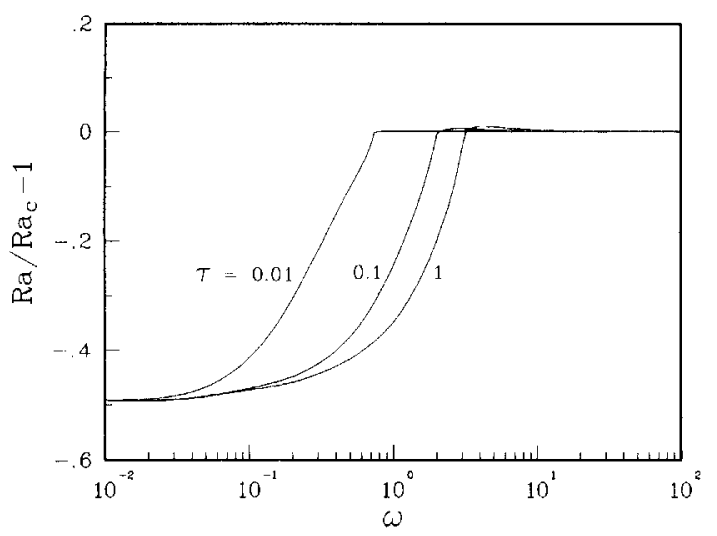

Fig. 5. Percentage change of critical Rayleigh number vs frequency at different optical thicknesses for $\operatorname{Pr}=0.7$, $B i=1, P=1$, and $\varepsilon=1$.

than for larger optical thicknesses and the maximum stabilization effect increases with increasing optical thickness. The percentage change of critical Rayleigh number can be used to understand the destabilization or stabilization of a modulated system compared with the unmodulated one. But the variation of the critical Rayleigh number with a radiative property is difficult to see unless the ordinate is expressed in terms of the absolute value. Figure 6 shows the variation of critical Rayleigh number vs optical thickness for different frequencies, with $\operatorname{Pr}=0.7, B i=1, P=1$, and $\varepsilon=1$. The curve for $\omega=10$ is known from the foregoing figures to have a slight stabilization effect, and the critical Rayleigh number is almost equal to the unmodulated case. For lower frequencies, it is seen that the lowest critical Rayleigh number occurs at a different optical thickness for each frequency, which is not seen for the unmodulated one. It can also be seen that the smaller the optical thickness, the lower the frequency that stabilization starts to occur.

Biot number was found to play dual roles on the occurrence of instability [2]. Decreasing the Biot number slightly decreases the critical Rayleigh number at large Biot numbers, and significantly increases the

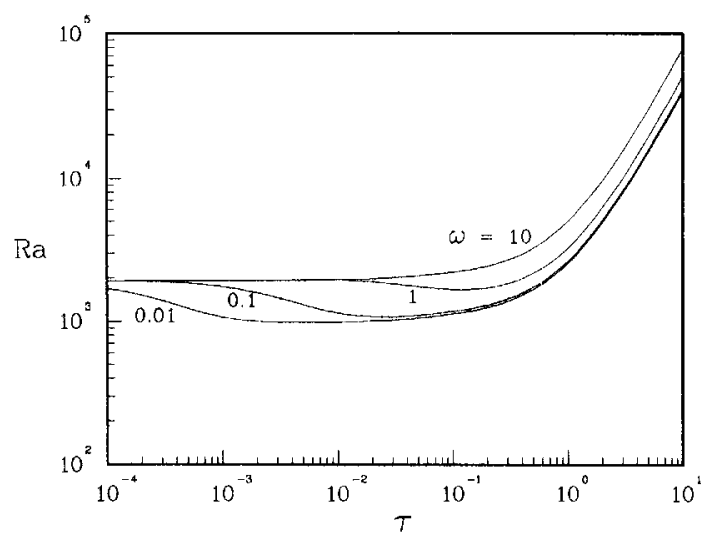

Fig. 6. Critical Rayleigh number vs optical thickness at different frequencies for $P r=0.7, B i=1, P=1$, and $\varepsilon=1$.

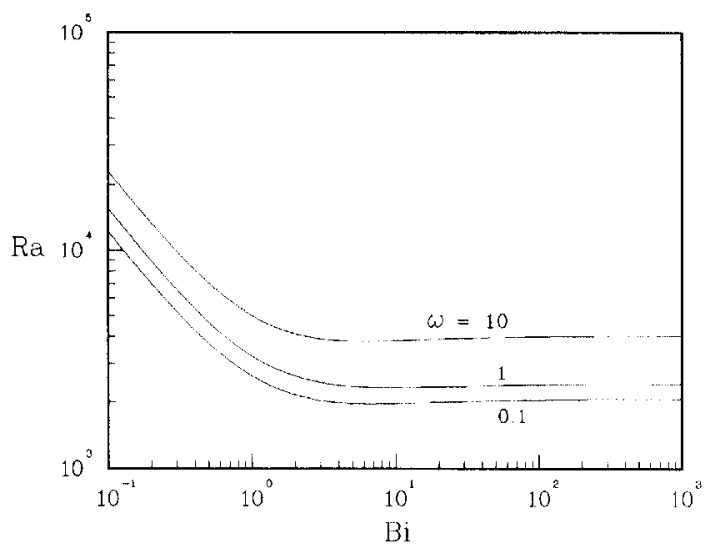

Fig. 7. Critical Rayleigh number vs Biot number at different frequencies for $\operatorname{Pr}=0.7, P=1, \tau=1$, and $\varepsilon=1$.

critical Rayleigh number at small Biot numbers. The effect of Biot number on the critical Rayleigh number at different frequencies is shown in Fig. 7 for $\operatorname{Pr}=0.7$, $P=1, \tau=1$ and $\varepsilon=1$. It is seen that the dependence of the critical Rayleigh number on the Biot number is similar for all frequencies. The rapid increase of critical Rayleigh number at small Biot number is due to the decrease of temperature difference between the lower plate and the upper surface.

\section{CONCLUSION}

When a fluid layer is sinusoidally heated from below, destabilization is found at low frequencies, and stabilization is found at high frequencies. In this study, a numerical method based on the linear stability theory and Floquet theory is developed to trace the stability boundary between stable and transiently stable states. The results are consistent with the amplitude criterion at low frequencies and periodicity criterion at high frequencies, developed by Rosenblat and Herbert [5], as well as the experimental data conducted by Finucane and Kelly [9].

When a fluid layer is heated by a modulated irradiation, energy absorbed by the lower boundary is released and heats the fluid. The mechanism is similar to when a fluid layer is heated by modulated heat from below. Consequently, the same destabilization effects are seen at low frequencies and stabilization at high frequencies, which increase with increasing modulation amplitude. Increasing the radiative parameters, such as Planck number and optical thickness, shifts the starting frequency of stabilization to a higher value and slightly increases the stabilizing effect at high frequencies.

Acknowledgement - The author would like to acknowledge the National Science Council, Republic of China for its support of the present work through project NSC 80-0401-E00919. 


\section{REFERENCES}

1. B. W. Webb and R. Viskanta, Radiation-induced buoyancy-driven flow in rectangular enclosures: experiment and analysis, $J$. Heat Transfer 109, 427-433 (1987).

2. W.-M. Yang, Thermal instability of a fluid layer induced by radiation, Numer. Heat Transfer 17, 365-376 (1990).

3. W.-M. Yang and M.-C. Leu, Instability of radiationinduced flow in an inclined slot, Int. J. Heat Mass Transfer 36, 3089-3098 (1993).

4. G. Venezian, Effect of modulation on the onset of thermal convection, J. Fluid Mech. 35, 243-254 (1969).

5. S. Rosenblat and D. M. Herbert, Low-frequency modulation of thermal instability, J. Fluid Mech. 43, 385-398 (1970).

6. S. Rosenblat and G. A. Tanaka, Modulation of thermal convection instability, Phys. Fluids 14, 1319-1322 (1971).

7. C.-S. Yih and C.-H. Li, Instability of unsteady flows of configurations-2. Convective instability, J. Fluid Mech. 54, 143-152 (1972)

8. P. M. Gresho and R. L. Sani, The effects of gravity modulation on the stability of a heated fluid layer, $J$. Fluid Mech. 40, 783-806 (1970).

9. R. G. Finucane and R. E. Kelly, Onset of instability in a fluid layer heated sinusoidally from below, Int. J. Heat Mass Transfer 19, 71-85 (1976).

10. R. J. Donnelly, Experiments on the stability of viscous flow between rotating cylinders-III. Enhancement of stability by modulation, Proc. R. Soc. Lond. A281, 130 139 (1964).

11. R. J. Donnelly, F. Reif and H. Suhl, Enhancement of hydrodynamic stability by modulation, Phys. Rev. Lett. 9, 363-365 (1962).

12. S. H. Davis, The stability of time-periodic flows, Ann. Rev. Fluid Mech. 8, 57-74 (1976).

13. V. S. Arpaci and D. Gozum, Thermal stability of radiating fluids: the Benard problem, Phys. Fluids 16, 581589 (1973).

14. E. L. Ince, Ordinary Differential Equations. Dover (1969).

15. E. A. Coddington and N. Levinson, Theory of Ordinary Differential Equations. McGraw-Hill, New York (1955).

16. L. Fox and I. B. Parker, Chebyshev Polynomials in Numerical Analysis. Oxford University Press, London (1968).

17. C. B. Moler and G. W. Stewart, An algorithm for generalized matrix eigenvalue problems, SIAM J. Numer. Anal. 10, 241-256 (1973). 